\title{
ON MODIFICATION OF SYMMETRIC RANK ONE FOR TRAINING NEURAL NETWORK BASED ON GRADIENT VECTOR
}

\author{
HASAN HAZIM JAMEEL ${ }^{*}$, and SALAh GHAZE SHAREEF ${ }^{* *}$ \\ *Dept. of Mathematics, College of Basic Education, University of Duhok, Kurdistan region - Iraq \\ ** Dept. of Mathematics, Faculty of Science, University of Zakho, Zakho, Kurdistan region - Iraq.
}

(Received: July 8, 2019; Accepted for Publication: November 28, 2019)

\begin{abstract}
In this paper, a modification of Symmetric Rank One (SR1) is propounded on the grounds of Modifying gradient-difference vector which meets Quasi condition and positive definite conditions. The new method is compared with the standard test results of the SR1 algorithm. In general, the modified method is more superior and efficient when compared to the standard Quasi-Newton method
\end{abstract}

KEY WORDS: SR1 method, Quasi-Newton method, Neural Network, optimization.

\section{INTRODUCTION}

A rtificial Neural Networks (ANNs) are greatly applied in various areas of science, in pattern classification, function approximation, optimization, pattern matching and associative memories [2,6]. Though the success and the promise of artificial neural networks in addressing practical problems, their design procedure still requires trial-and-The error. Attainment of the optimal structure of artificial neural networks for a problem is one of the typical problems in neural network design. Back propagation (BP) learning can recognize the training of feed-forward multilayer neural network. The algorithm primarily revises neural network weights pursuant to the gradient descent methods to decrease errors.

The ANN is comprised of a set of processing factors known as neurons or nodes which are interconnected with each other [1]. Usually, the node transfer function is a nonlinear function suchlike a sigmoid function, a Gaussian function, etc. In this study, sigmoid function is employed.

The optimization aim is to minimize the objective function by optimizing the network weight. $E(w)$ means that a square function is selected as a sum error function[10].

$E(w)=\frac{1}{2} \sum_{r=1}^{m} \sum_{l=1}^{p}\left(O_{l}^{p}-t_{r}^{m}\right)^{2}$ where $w$ is weight vector at each iteration; $O_{l}^{p}$ and $t_{r}^{m}$ represent respectively the actual and predicted value. For effectively training neural networks in scientific and engineering, SR1 algorithm is considered one of the most competitive formulations among the QuasiNewton methods which is known as one of the most efficient manners to solve nonlinear unconstrained or bound constrained optimization problems. These methods are mostly utilized when the second derivative matrix of the objective function is either unavailable or too pricey to compute, they allow. Therefore, the curvature of the problem to be exploited in the numerical algorithm, despite the fact that only first derivatives (gradients) and function values are required see $[4,5,11,12]$.

The Symmetric rank one produce the sequence of weight $\left\{w_{\mathrm{j}}\right\}$, is given by

$w_{j+1}=w_{j}+\delta_{j} d_{j}$

Where the search direction is and satisfies the descent condition

$d_{j}=-H_{j} \nabla E\left(w_{j}\right) j>0$

$H_{j}$ is usually required to be positive definite to assure a descent direction for $E . H_{j}$ is upgraded at each iteration using gradient vector, and $\delta_{j}>0$ is learning rate in machine learning. The $H_{j}$ is defined using sequences of vectors $s_{j}$ and $y_{j}$, which are given as

$s_{j}=w_{j+1}-w_{j}$ and $y_{j}=\nabla E\left(w_{j+1}\right)-\nabla E\left(w_{j}\right)$ (1.4) 
The SR1 is the unique rank-one in the class of Quasi Newton update satisfying QuasiNewton condition:

$H_{j+1} y_{j}=s_{j}$

where $H_{j+1}=H_{j}+\frac{\left(s_{j}-H_{j} y_{j}\right)\left(s_{j}-H_{j} y_{j}\right)^{T}}{y_{j}^{T}\left(s_{j}-H_{j} y_{j}\right)}$,

where $y_{j}$ and $s_{j}$ are defined in (1.4) and

$y_{j}^{T}\left(s_{j}-H_{j} y_{j}\right) \neq 0$ for each $j$, see $[4,7]$.

Proving the positive definite condition of Quasi-Newton method, SR1, usually needs that the step size of $\delta_{j}$ achieves the following Wolfe Conditions [13].

$E\left(w_{j}+\delta_{j} d_{j}\right) \leq E\left(w_{j}\right)+\sigma_{1} \delta_{j} \nabla E\left(w_{j}\right)^{T} d_{j}$ (1.7)

$\left|\nabla E\left(w_{j}+\delta_{j} d_{j}\right)^{T} d_{j}\right| \leq \sigma_{2}\left|\nabla E_{j}^{T} d_{j}\right|$

where $0<\sigma_{1}<\sigma_{2}<1$.

So, the standard Wolfe condition is (1.7) and

$\nabla E\left(w_{j}+\delta_{j} d_{j}\right)^{T} d_{j} \geq \sigma_{2} \nabla E_{j}^{T} d_{j}$

This study is outlined as follows: in section one, we present an introduction to neural network. Section Two sheds light on SR1 modification whereas the third one deals with the proof of quasi and positive definite condition which verifies the SR1 modification. The forth section illustrates the numerical results which are compared with standard test results.

\section{SYMMETRIC RANK ONE MODIFICATION}

In this section, modified SR1 is suggested for modifying SR1 by the usage of gradientdifference vector given in (1.6)

$\overline{y_{J}}=y_{j}+(1-\theta)\left(G_{j} s_{j}-y_{j}\right)$ where $0<\theta<1$ (2.1)

For more details see [8].

In a bid to use the Hessian in $H_{j}$ Andrei in [9] suggested a nonlinear conjugate gradient algorithm in which the Hessian/vector product $\nabla^{2} E\left(w_{j+1}\right) s_{j}$ is approximated by finite differences:

$\overline{y_{J}}=y_{j}+(1-\theta)\left(\frac{y_{j}}{\sigma}-y_{j}\right)$

where $\sigma=\frac{2 \sqrt{\epsilon_{m}}\left(1+\left\|w_{j+1}\right\|\right)}{\left\|s_{j}\right\|}$, and $\epsilon_{m}$ is error machine used for accuracy which is the smallest positive $<1$.

Thus

$$
H_{j+1}^{\text {new }}=H_{j}+\frac{\left(s_{j}-H_{j} \bar{y}_{j}\right)\left(s_{j}-H_{j} \bar{y}_{j}\right)^{T}}{\bar{y}_{j}^{T}\left(s_{j}-H_{j} \bar{y}_{j}\right)}
$$

Algorithm 1.

Step (1) Let $w_{0}$, an initial point, be given as well as an identity nxn symmetric positive definite $H_{0}, \varepsilon$ is a termination scalar and set $j=$ 0 .

Step (2) compute $d_{j}=-H_{j} g_{j}$ where $g_{j}=$ $\nabla E\left(w_{j}\right)$.

Step (3) Calculate $\delta_{j}$ to minimize $E\left(w_{j}+\delta_{j} d_{j}\right)$.

Step (4) find new point of weight from (1.2)

Step (5) If $\left\|g_{j+1}\right\|<\varepsilon$ then $w^{*}=w_{j}$ then stop Else find $s_{j}$ from $s_{j}=w_{j+1}-w_{j}$ go to

step (5)

Step (6) Evaluate the hessian matrix by using (2.2) and (2.3).

Step (7) set $j=j+1$ go to step 2 .

\section{QUASI AND POSITIVE DEFINITE CONDITIONS}

In this section, we have proved that the modification of SR1 is satisfying Quasi and positive definite conditions.

Theorem3.1 If the new algorithm is applied to the quadratic with Hessian $G=G^{T}$, then $H_{j+1} \bar{y}_{j}=s_{j}, j>0$.

Proof: Multiplying both sides of (2.3) by $\bar{y}_{j}$ from right, we have:

$H_{j+1}^{\text {new }} \bar{y}_{j}=H_{j} \bar{y}_{j}+\frac{\left(s_{j}-H_{j} \overline{y_{j}}\right)\left(s_{j}-H_{j} \overline{y_{j}}\right)^{T}}{\bar{y}_{j}^{T}\left(s_{j}-H_{j} \overline{y_{j}}\right)} \bar{y}_{j}$

(3.2)

It is clear that $\left(s_{j}-H_{j} \bar{y}_{j}\right)^{T} \bar{y}_{j}$ is scalar and $\bar{y}_{j}^{T}\left(s_{j}-H_{j} \bar{y}_{J}\right)$ is also scalar $\therefore\left(s_{j}-H_{j} \bar{y}_{j}\right)^{T} \bar{y}_{j}=\bar{y}_{j}^{T}\left(s_{j}-H_{j} \bar{y}_{j}\right)$

(3.3)

Therefore we have

$H_{j+1}^{n e w} \bar{y}_{j}=H_{j} \bar{y}_{j}+\left(s_{j}-H_{j} \bar{y}_{j}\right)$

(3.4)

$H_{j+1}^{n e w} \overline{y_{j}}=s_{j}$

Theorem3.2 If $H_{j}$ is a positive definite, then the matrix $H_{j+1}$ generated by the $H_{j+1}^{\text {new }}$ algorithm is also positive definite.

Proof: Multiplying both sides of (2.3) by $\bar{y}_{j}$ from right and by $\bar{y}_{j}{ }^{T}$ from left, we have 


$$
\begin{aligned}
\bar{y}_{j}{ }^{T} H_{j+1}^{n e w} \bar{y}_{J}= & \bar{y}_{J}^{T} H_{j} \bar{y}_{J} \\
& +\frac{\bar{y}_{J}^{T}\left(s_{j}-H_{j} \bar{y}_{j}\right)\left(s_{j}-H_{j} \bar{y}_{J}\right)^{T} \bar{y}_{j}}{\bar{y}_{j}{ }^{T}\left(s_{j}-H_{j} \bar{y}_{J}\right)}
\end{aligned}
$$

So

$$
\bar{y}_{j}^{T} H_{j+1}^{n e w} \bar{y}_{J}=s_{j}^{T} \overline{y_{J}}
$$

By substituting (2.2) in (3.5) we get

$\bar{y}_{J}^{T} H_{j+1} \bar{y}_{J}=s_{j}^{T}\left[\left(y_{j}+(1-\theta)\left(\frac{y_{j}}{\sigma}-y_{j}\right)\right]\right.$,

where $0<\theta<1$.

$$
=s_{j}^{T} y_{j}\left[1+(1-\theta)\left(\frac{1}{\sigma}-1\right)\right]
$$$$
\text { Suppose that } \mathrm{k}=\left[1+(1-\theta)\left(\frac{1}{\sigma}-1\right)\right]
$$

Because $\sigma$ is between $0 \& 1$ so $k$ will always be greater than zero.

$$
\begin{aligned}
s_{j}^{T} y_{j} & =s_{j}^{T}\left(\nabla E\left(w_{j+1}\right)-\nabla E\left(w_{j}\right)\right) \\
& =s_{j}^{T} \nabla E\left(w_{j+1}\right)-s_{j}^{T} \nabla E\left(w_{j}\right) \\
& =\alpha_{j} d_{j}^{T} \nabla E\left(w_{j+1}\right)+\alpha_{j} \nabla E\left(w_{j}\right)^{T} H_{j} \nabla E\left(w_{j}\right)
\end{aligned}
$$

By using Wolfe condition in (1.9)

$s_{j}^{T} y_{j} \geq \alpha_{j} \sigma_{2} d_{j}^{T} \nabla E\left(w_{j}\right)+\alpha_{j} \nabla E\left(w_{j}\right)^{T} H_{j} \nabla E\left(w_{j}\right)$

$$
=-\alpha_{j} \sigma_{2} \nabla E\left(w_{j}\right)^{T} H_{j} \nabla E\left(w_{j}\right)+
$$

$\alpha_{j} \nabla E\left(w_{j}\right)^{T} H_{j} \nabla E\left(w_{j}\right)$, where $0<\sigma_{2}<1$

$$
=\left(1-\sigma_{2}\right) \alpha_{j} \nabla E\left(w_{j}\right)^{T} H_{j} \nabla E\left(w_{j}\right)
$$

Since $0<\sigma_{2}<1$, and $H_{j}$ is positive definite

Then, $\left(1-\sigma_{2}\right) \alpha_{j} \nabla E\left(w_{j}\right)^{T} H_{j} \nabla E\left(w_{j}\right)>0$

$s_{j}^{T} y_{j}>0$.

$\therefore s_{j}^{T} y_{j}\left[1+(1-\theta)\left(\frac{1}{\sigma .}-1\right)\right]>0$.

So $\bar{y}_{J}^{T} H_{j+1} \bar{y}_{J} \geq 0$

\subsection{Numerical Results}

This section is devoted to testing the implementation of the modified methods. The modified method is compared to the standard SR1. The results given in Table 1 specifically quote the NOI and NOF. Table 1 shows that the modified SR1 method is superior to standard

\begin{tabular}{|c|c|c|c|c|c|}
\hline \multirow[t]{2}{*}{ Test Function } & \multirow[t]{2}{*}{$\mathbf{N}$} & \multicolumn{2}{|c|}{ Standard formula SR1 } & \multicolumn{2}{|c|}{ MODIFIED SR1 } \\
\hline & & $\mathrm{NOI}$ & NOF & $\mathrm{NOI}$ & NOF \\
\hline \multirow[t]{5}{*}{ G-Central } & 4 & 36 & 253 & 15 & 90 \\
\hline & 100 & 43 & 331 & 29 & 215 \\
\hline & 500 & 60 & 496 & 32 & 247 \\
\hline & 1000 & 66 & 554 & 49 & 411 \\
\hline & 5000 & 72 & 616 & 63 & 549 \\
\hline \multirow[t]{7}{*}{ Miler } & 4 & 34 & 329 & 26 & 105 \\
\hline & 100 & 47 & 182999 & 43 & 938 \\
\hline & 500 & 53 & 183098 & 51 & 170 \\
\hline & 1000 & 53 & 183098 & 48 & 162 \\
\hline & 5000 & 65 & 189123 & 57 & 203 \\
\hline & 4 & 31 & 90 & 30 & 81 \\
\hline & 100 & 32 & 94 & 30 & 81 \\
\hline \multirow[t]{5}{*}{ Rosen } & 500 & 33 & 98 & 30 & 81 \\
\hline & 1000 & 37 & 115 & 30 & 80 \\
\hline & 5000 & 37 & 120 & 31 & 84 \\
\hline & 4 & 11 & 24 & 11 & 24 \\
\hline & 100 & 44 & 89 & 44 & 89 \\
\hline \multirow[t]{3}{*}{ GWolfe } & 500 & 47 & 95 & 47 & 95 \\
\hline & 1000 & 50 & 101 & 49 & 99 \\
\hline & 5000 & 106 & 294 & 105 & 212 \\
\hline \multirow[t]{4}{*}{ Cubic } & 4 & 15 & 48 & 12 & 34 \\
\hline & 100 & 16 & 66 & 16 & 46 \\
\hline & 500 & 16 & 51 & 16 & 46 \\
\hline & 1000 & 16 & 55 & 16 & 46 \\
\hline
\end{tabular}
(SR1) method with respect to NOI and NOF. Furthermore, the modified SR1 algorithms and the standard SR1 algorithms are compared when the input $p=\left[\begin{array}{ll}0.1 & 0.1\end{array}\right]$ and target $t=\left[\begin{array}{ll}1 & 1\end{array}\right]$. The target error has been set to 0.01 and the maximum epochs to 3000 . The numerical results can be seen in Table 3.

Table (1): Comparison between Modified and Standard SR1 Algorithm 


\begin{tabular}{llllll}
\hline & 5000 & 16 & 50 & 16 & 46 \\
& & & & & \\
\hline Gpowell3 & 4 & & & 13 & 31 \\
& 100 & 14 & 35 & 14 & 33 \\
& 500 & 15 & 37 & 15 & 35 \\
& 1000 & 15 & 37 & 15 & 35 \\
\hline sum & 5000 & 15 & 37 & 15 & 35 \\
& 4 & 3 & 40 & 3 & 11 \\
& 100 & 14 & 11 & 21 & 80 \\
& 500 & 21 & 119 & 23 & 121 \\
& 1000 & 23 & 123 & 38 & 176 \\
\hline Total & 5000 & 38 & 176 & 1067 & 4909 \\
& & 1210 & 742985 & & \\
\hline
\end{tabular}

Table (2): The Rate of Improvement between Modified Algorithm and Standard SR1 algorithm.

\begin{tabular}{lcc}
\hline zTools & SR1 & Modified SR1 \\
\hline NOI & $100 \%$ & 88.1818 \\
\hline NOF & $100 \%$ & 0.6607 \\
\hline
\end{tabular}

The above table illustrates the rate of improvement in the modified standard SR1 algorithm. The numerical results of the new algorithm are better than the standard algorithm. As noted, the number of iterations and the number of function evaluations of the standard algorithm are about $100 \%$. In other words, the new algorithm has improvement as compared to standard algorithm with $11.8182 \%$ in NOI and $99.3393 \%$ in NOF when $\theta \in(0,1)$.

Table (3): Comparing the Performance of Modified Algorithm with Standard SR1 Algorithm.

\begin{tabular}{lll}
\hline Methods & No. & Epochs \\
& Running & \\
\hline SR1 & 1 & 1000 \\
& 2 & 1000 \\
& 3 & 833 \\
& 4 & 1000 \\
& 5 & 1000 \\
\hline Modified & 1 & 677 \\
& 2 & 165 \\
& 3 & 268 \\
& 4 & 224 \\
& 5 & 1000 \\
\hline
\end{tabular}




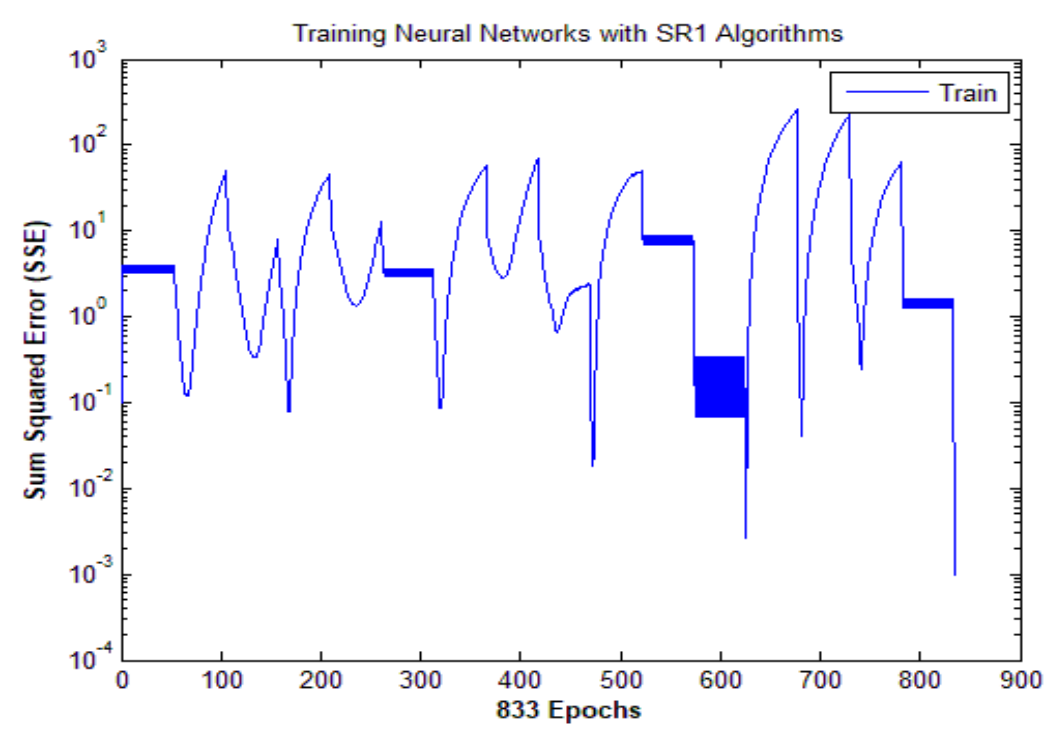

Fig.(1): Performance of standard SR1 algorithm for training neural networks.

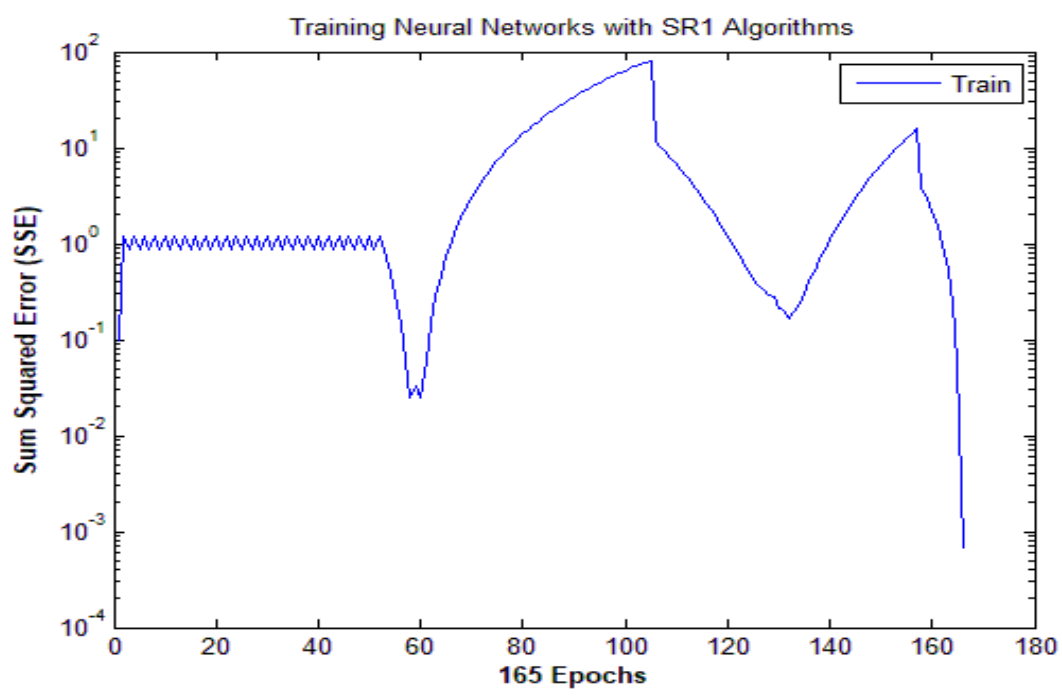

Fig. (2): Performance of modified SR1 algorithm for training neural networks.

\section{CONCLUSION}

This work, propounded a modification of SR1 by using gradient-difference vector. The quasi-newton condition and positive definite have been proved. In addition, the modification algorithms are used for training neural networks according to outcomes a modified method is more superior and effective than the standard SR1.

\section{REFERENCES}

$\mathrm{X}$. Yao, Evolving artificial neural networks, Proceedings of the IEEE, 1999, vol. 87, no. 9, pp. 1423-1447,.
J. Dayhoff, An Introduction to Neural Network Architectures, New York: Van Nostrand Reinhold, 1990.

J. Nocedal and S. J. Wright. Numerical Optimization. Springer, New York, 2006, 2nd edition.

J.E. Dennis and J.J. Mor6, Quasi-Newton methods, motivation and theory, SIAM Review 1977, 19, 46-89.

J.E. Dennis and R.B. Schnabel, Numerical Methods for Unconstrained Optimization and Nonlinear Equations, Prentice-Hall, Englewood Cliffs, NJ, 1983, 4. 
K. Mehrotra, C. K. Mohan, and S. Ranka, Elements of Artificial Neural Networks, Cambridge, MA: MIT Press, 1997.

L. Bottou, F. Curtis, and J. Nocedal. Optimization methods for large-scale machine learning, SIAM Review, 2018, 60(2):223311.

M. Al-Baali and H. Khalfan, An Overview of Some Practical QuasiNewton Methods for Unconstrained Optimization, SQU Journal For Science, 2007, 12 (2) 199-209.

N. Andrei, Accelerated conjugate gradient algorithm with finite difference Hessian/vector product approximation for unconstrained optimization, J. Comput. Appl. Math, 2009, 230, no. 2, 570-582.
Ngoc Tam Bui and Hiroshi Hasegawa, Training Artificial Neural Network Using Modification of Differential Evolution Algorithm, International Journal of Machine Learning and Computing, February 2015, Vol. 5, No. 1 .

P.E. Gill, W. Murray and M.H. Wright, Practical Optimization Academic Press, New York, 1981,8

R. Fletcher, Practical Methods of Optimization: Unconstrained Optimization, Wiley, Chichester, 1980.

Stephen J. Wright and Jorge Nocedal, numerical optimization. Springer new york 2006. 